\title{
Practical obstacles and their mitigation strategies in compressional optical coherence elastography of biological tissues
}

\author{
Vladimir Y. Zaitsev* ${ }^{* \dagger}$, , Alexandr L. Matveyev*,†, Lev A. Matveev*, ${ }^{*, \dagger}$ \\ Ekaterina V. Gubarkova ${ }^{\dagger}$, Alexandr A. Sovetsky*, Marina A. Sirotkina ${ }^{\dagger}$, \\ Grigory V. Gelikonov*,†, Elena V. Zagaynova ${ }^{\dagger}$, Natalia D. Gladkova ${ }^{\dagger}$ \\ and Alex Vitkin ${ }^{\dagger}+$ \\ *Institute of Applied Physics \\ Russian Academy of Sciences \\ Ulyanova Street 46 \\ Nizhny Novgorod 603950, Russia \\ ${ }^{\dagger}$ Nizhny Novgorod State Medical Academy \\ 10/1 Minina Square \\ Nizhny Novgorod 603005, Russia \\ *University of Toronto and University Health Network \\ 610 University Avenue, Toronto \\ ON M5G 2M9, Canada \\ §vyuzai@ipfran.ru
}

Received 29 May 2017

Accepted 13 August 2017

Published 29 September 2017

\begin{abstract}
In this paper, we point out some practical obstacles arising in realization of compressional optical coherence elastography (OCE) that have not attracted sufficient attention previously. Specifically, we discuss (i) complications in quantification of the Young modulus of tissues related to partial adhesion between the OCE probe and soft intervening reference layer sensor, (ii) distorting influence of tissue surface curvature/corrugation on the subsurface strain distribution mapping, (iii) ways of signal-to-noise ratio (SNR) enhancement in OCE strain mapping when periodic averaging is not realized, and (iv) potentially significant influence of tissue elastic nonlinearity on quantification of its stiffness. Potential practical approaches to mitigate the effects of these complications are also described.
\end{abstract}

Keywords: Optical coherence elastography; optical coherence tomography; phase-resolved methods; strain mapping; stiffness characterization.

$\S_{\text {Corresponding author. }}$

This is an Open Access article published by World Scientific Publishing Company. It is distributed under the terms of the Creative Commons Attribution 4.0 (CC-BY) License. Further distribution of this work is permitted, provided the original work is properly cited. 


\section{Introduction}

Elastography (i.e., characterization of mechanical properties of biological tissues, in particular, evaluation of their shear modulus $G$ responsible for ability of tissues to maintain their form) is a diagnostic imaging modality that complements conventional structural bio-imaging. Initially, it has been proposed and realized in medical ultrasound, ${ }^{1}$ where "classical" structural images do not directly visualize even quite significant variations in the tissue stiffness. This is explained by very weak influence of the shear modulus on the velocity $V_{p}=[(K+4 G / 3) / \rho]^{1 / 2}$ of longitudinal ultrasound waves, because in soft biological tissues, this velocity is mostly determined by the bulk modulus $K$ and tissue density $\rho .^{2}$ Indeed, in most soft biological tissues, the shear modulus is orders of magnitude smaller than the bulk modulus, $G \ll K$, such that the effects of variations in modulus $G$ on propagation of ultrasound are diminishingly small. Although the shear modulus may differ significantly (sometimes orders of magnitude) for different tissues or different states of the same tissue, the condition $G \ll K$ remains valid and direct influence of modulus $G$ on ultrasound wave velocity $V_{p}$ remains hardly noticeable.

In view of this, ultrasonic bio-imaging of the shear modulus requires either utilization of ultrasonic waves to visualize additionally excited shear waves (for which the velocity $V_{s}=[G / \rho]^{1 / 2}$ is directly related to modulus $G$ ), or alternatively can be based on comparison and specialized processing of conventional ultrasonic scans of reference and deformed tissue images in dynamic or quasi-static regime. The latter approach is called "compressional elastography", where approximately uniaxial (one-directional) stress application and advanced signal processing comparing pre-and postdeformation scans are used to characterize the Young modulus E. For soft biological tissues with $G \ll K$, the relationship $G=E / 3$ holds with a high accuracy. Since moduli $G$ and $E$ are proportional to each other, they can be equally used to characterize the "stiffness" of tissues, i.e., the ability to maintain their form. This ability contrasts with the behavior of liquids that strongly resist all-round compression, but have zero shear modulus and cannot keep form.

In a similar manner, MRI techniques can also be used for the visualization of auxiliary quasistatic or dynamic deformations of biological tissues for characterizing spatial distribution of their stiffness. ${ }^{1}$

By analogy with other elastographic methods, several variants of elastographic imaging have been proposed in optical coherence tomography (OCT) (see Refs. 3-9), where auxiliary dynamic or quasistatic tissue deformations are optically visualized. One possibility is to excite a surface of a shear wave and use OCT for visualizing the wave propagation. Then, the surface/shear wave velocity can be related to the shear elastic modulus $G$ via a known theoretical relationships ${ }^{10-12}$ based only on the measuring displacements in the propagating wave without the necessity of making additional measurements of stress, which is a very attractive feature of the method. This approach may prove feasible in vivo, for example, in ophthalmologic applications. As a possible limitation, this method requires access to the open surface of the tissue and the use of an additional device (e.g., a miniature air gun) for exciting the surface wave. Similarly, focused high-frequency ultrasound beams can be used for exciting either surface or bulk shear waves with visualization of their propagation by means of OCT. ${ }^{13,14}$

Alternatively, excised tissue can be placed on an actuator producing oscillations with known amplitude and frequency (and therefore known as inertia force). Then, by measuring resultant amplitude of tissue oscillations, its Young modulus $E$ can be evaluated. ${ }^{15}$ Such an approach may be limited in its application to the use of excised samples.

Another attractive approach that has been actively pursued in OCT since the seminal work of Schmitt ${ }^{16}$ is compressional optical coherence elastography (OCE). Similar to the ultrasound discussed above, here the comparison is made between OCT scans of the tissue in its initial and deformed states. The tissue deformation can be produced by the output window of the OCT probe, so that this principle can be realized both ex vivo and in vivo (if accessible by the OCT probe). The deformation can be created either using a special (e.g., piezo-) actuator $^{9,17}$ or produced manually using a handheld OCT, with pros/cons for both approaches. The use of a special actuator makes it possible to produce periodic stable excitation and perform temporal averaging for enhancing effective signalto-noise ratio (SNR). ${ }^{18}$ This may be directly applicable when studying excised samples, and 
potentially beyond them. The use of manual compression is more attractive due to its simplicity and easier applicability in vivo, although other ways of enhancing SNR rather than periodic averaging need to be developed (some are discussed below).

Two decades after the initial formulation of the basic compressional OCE concepts,${ }^{16}$ the problem of strain reconstruction by comparing reference and deformed scans appears to be solved (instead of initially proposed correlational principles, phasesensitive approaches have proven more practi$\left.\mathrm{cal}^{17,19}\right)$. However, the expectation that simple visualization of strains throughout the OCE image would be sufficient to draw unambiguous conclusions about tissue stiffness distribution (often approximated by a plane-layered structure) appears to be oversimplified. This expectation implies several simplifying assumptions such as a simple linear relationship between strain and stress, and a priori uniform uniaxial strain distribution in the vicinity of the compressing OCT-probe window or a special compressing piston.

However, recent studies of compressional OCE realizations have indicated that real tissue situations can be considerably more complex, with potential significant implications for bio-OCE accuracy (for example, see Refs. 20-24 and the discussion below). Among these works, Doyley ${ }^{20}$ described the ultrasound-based elastography, where the main elastographic approaches use similar tissue deformations (quasi-static and dynamic) as mentioned above; the reconstructed quasi-static or dynamic strains are used for obtaining information about the tissue stiffness distributions. In this context, Ref. 20 is focused on the utilization of advanced numerical methods for finding the connection between the initial reconstructed displacement/strain fields and the stiffness distribution by minimizing the difference between the measured and model-predicted displacements/strains. Such numerical approaches look particularly suitable for bio-materials with significant (lateral) inhomogeneities in stiffness, for which simpler algebraic solutions connecting strain and stiffness may lead to significant inaccuracy. Recent works ${ }^{23,24}$ discuss the application of similar numerical approaches in OCE for improving the accuracy of stiffness quantification. However, incorrectly measured strains may result in significant inaccuracy of stiffness estimates, which may be important for both simple algebraic solutions relating strain and stress, and for advanced numerical approaches similar to those used in Refs. 20, 23 and 24. Bearing this in mind in the following sections, we discuss somewhat different OCE aspects, first of all, certain features/practical obstacles in experimental reconstruction of strains in compressional OCE.

\section{State-of-the-Art in Compressional OCE: Potential Obstacles and Their Mitigation}

In this section, we point out some practical obstacles arising in the realization of compressional OCE that have not drawn due attention previously, and discuss the ways of their resolution. Specifically we discuss (i) some complications in using soft intervening reference layers ("compliant sensors" 17 ) for quantification of the Young modulus of underlying tissues, (ii) the distorting influence of tissue surface curvature/corrugation on strain distribution in the tissue bulk, (iii) ways of enhancement of effective SNR in OCE strain mapping when periodic averaging is not possible, and (iv) potential significant influence of elastic nonlinearity of biological tissue quantification of its stiffness even in low-strain regimes where fairly linear tissue response is intuitively expected.

In what follows, we limit ourselves to the discussion of physical aspects of the measurement procedures, and of applying simple and practical algebraic approaches for the inverse problem of relating the measured strains to the elastic modulus, although numerical (finite element) approaches can also be very useful for this purpose. ${ }^{20,23,24}$ Iterative procedures to enable best correspondence between the measured and predicted strain distributions can be particularly apt when the stiffness heterogeneities have pronounced variations in the lateral direction, exhibiting sharp edges and high stiffness contrast; however, such situations are not very common in soft tissues. That is, in many biological materials, the transition between different stiffness zones is fairly gradual to be satisfactorily approximated by layered structures with smooth lateral variations. Thus, simple first-order algebraic relationship between strains and stiffness distributions appears suitable. Since this is biomedically realistic and considerably less demanding computationally, in what follows, we limit ourselves to such 
straightforward relationship between strain and stiffness distributions.

\subsection{Adhesion effects in using intervening sensor layers for quantifying underlying tissue stiffness}

As mentioned in the introduction, the main objective of elastography is the evaluation of shear modulus $G$ of soft biological tissues, although in the literature, the Young modulus $E$ is also equivalently used. This equivalent usage is possible bearing in mind that for soft tissues, the shear modulus $G$ with typical values $G \sim 10^{4}-10^{6} \mathrm{~Pa}$ is much smaller than the bulk modulus $K$ that is dominated by compressibility of water with $K \approx 2.25 \cdot 10^{9} \mathrm{~Pa}^{25}$ (the water content of many soft biological tissues is $\sim 70 \%$ or greater, which thus dominates both their compressibility and density). Since $G / K \sim 10^{-5}-10^{-3} \ll 1$ for all soft tissues, the Poisson's ratio $\nu=(3-2 G / K) /(6+2 G / K) \rightarrow 0.5$, i.e., remains close to $\nu=0.5$ typical of liquids. In view of the relationship $G=E / 2(1+\nu),{ }^{2}$ for $\nu \rightarrow 0.5$, the shear modulus $G$ is nearly proportional to the Young modulus $E$ (i.e., $G \approx E / 3$ ). Thus, both moduli $G$ and $E$ can be equivalently used to characterize the soft-tissue shear stiffness (to within a factor of $3 \times$ ).

If the tissue can be approximated as a planelayered structure that is compressed by a rigid OCT probe and the tissue can freely move laterally (i.e., assuming no probe-sample adhesion ("stiction")), then near the rigid-probe surface, the mechanical stress $\sigma$ is approximately uniform and constant, and has only one axial component. ${ }^{26}$ Then, the ratio between axial strains of the layers is inversely proportional to the ratio of the elastic moduli $E$ and $G$, and

$$
\frac{\varepsilon_{1}}{\varepsilon_{2}}=\frac{E_{2}}{E_{1}}=\frac{G_{2}}{G_{1}},
$$

where $\varepsilon_{1,2}$ are strains of the layers. Linear relationship (1) is based on Hooke's law $\sigma=\varepsilon \cdot E$, and means that axial strain distribution produced by the uniaxial one-directional compression of the OCT probe can also yield the distribution of the tissue compliance, i.e., inverse stiffness (moduli $G^{-1}$ or $\left.E^{-1}\right)$. We can now ask whether compressional OCE can yield only relative images of the various derived elastographic quantities of interest, or whether (and how) quantitative absolute values determination is possible.

Direct quantification of the applied stress (which is needed to recalculate the visualized strains into the Young modulus as $E=\sigma / \varepsilon$ ) is a nontrivial problem that requires sufficiently accurate estimation of force applied by the OCT probe onto the studied tissue region with a known area (see e.g., Ref. 22 where the tissue is imaged through a Fabry-Perot resonator that makes it possible to estimate the variations in the resonator thickness and thus calculates the applied force). Fortunately, instead of directly measuring stress, the same Eq. (1) suggests that one can quantify the stiffness distribution throughout the imaged region if the elastic modulus is a priori known for one of the visualized layers. Such a reference layer (made from a translucent material (e.g., silicone) with a known/independently measured elastic modulus) can be placed between the OCT probe and the studied tissue, and serves as a calibration sensor as shown in Fig. 1(a). Thus, the ratio of strains in the reference layer and tissue yields the ratio of their

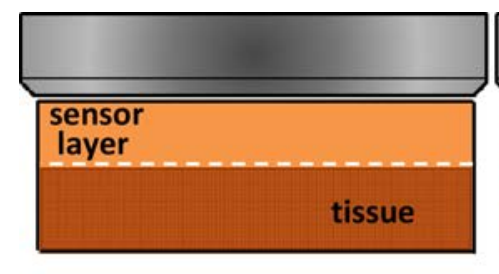

(a)

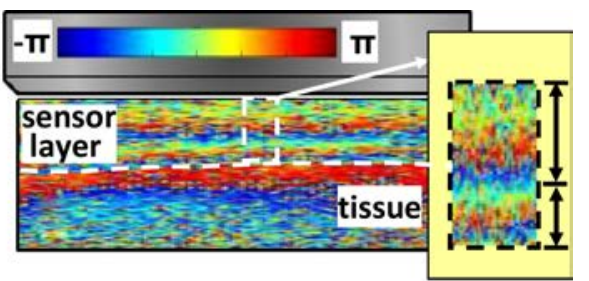

(b)

Fig. 1. The use of independently-calibrated sensor layer to study underlying tissue in compressional OCE, and associated real-life artefacts. (a) idealized plane-parallel geometry showing the compressional probe, sensor layer, and interrogated tissue that can freely expand laterally; (b) an experimental example of interframe OCE phase distribution, with the dashed line showing the actual interface between the sensor layer (homogeneous silicone) and tissue (tumor inoculated in the mouse ear); the inset zooms in on a region of phase gradient heterogeneity in the homogeneous sensor layer caused by its partial sticking to the OCT probe. 
inverse Young moduli. In fact, this idea of the use of reference layer was proposed in Ref. 27 for ultrasound-based compressional approach, although it has not found wide application in medical ultrasound elastography.

In OCE, such an approach has been pioneered by the group of Sampson et al. ${ }^{17,28}$ where the referencelayer strain $s_{\text {ref }}$ is estimated by measuring its thickness $l(x, y)$ in normal and axially-compressed states via $s_{\text {ref }}=\left(l-l_{0}\right) / l_{0}$. Inside the tissue itself, the strain is determined by comparing pixel phases in the OCT scans for the initial and deformed states and then computing the vertical gradient of the interframe phase difference using a weighted leastsquare procedure. ${ }^{29}$ We recently proposed an alternate approach to estimate the compression phase gradients called the "vector method" 30,31 that also draws upon the OCE measurements with the overlying reference layer. The common and attractive feature of both realizations ${ }^{17,31}$ is that they do not require the knowledge of the tissue refractive index. The advantage of the vector method is its robustness with respect to various noises, so that for the chosen size of the processing window, it can outperform the least-square method of gradient estimation even if the latter is used with amplitude weighting to reduce contribution of noisy weakamplitude pixels (see details in Ref. 31); its additional advantage is obviating the necessity of phase unwrapping even if wrapping occurs within the vertical size of the processing window. Furthermore, the vector method ${ }^{31}$ of strain estimation within the translucent reference layer does not require visualization of its entire thickness, thus sparing much of the useful visualized area in the OCT image for tissue itself.

The ability of this procedure to estimate local strains in both tissue and reference layers may be useful to minimize the distorting influence of the boundary conditions (e.g., adhesion or stiction) at the interface between the OCT probe and the reference layer. Indeed, the reference layer strain near its contact with a rigid boundary may be significantly reduced. This is explained by partial sticking of the soft reference layer to the probe, which impedes ideal free expansion of the material in lateral directions and thus distorts the apparent Young modulus of the calibration material in the near-interface region. This is demonstrated in Fig. 1 (b) by an example of inter-frame phase distribution inside a reference layer (silicone) and underlying tissue (tumor in mouse ear). The dashed curve corresponds to the layer-tissue interface, and the inset shows a zoomed fragment of the homogeneous silicone in which the effect of partial sticking causes noticeably inhomogeneous vertical phase gradient. The inset shows that the gradient of color variation is noticeably reduced near the layer-probe interface due to the influence of adhesion. This effect precludes the use of thin reference layers (in practice $<50 \mu \mathrm{m}$ ), otherwise the apparent strain and the corresponding effective modulus may be very strongly distorted by adhesion effects at the interface. However, for a given lateral size of the tissuecompressing piston and other conditions of the compression, it is possible to use a somewhat thicker layer (say, $\sim 150-200 \mu \mathrm{m}$ ), so that in the deeper part of the layer, the strain distribution would become more uniform. It is also possible to visualize only its lower part (where the top boundary influence is noticeably reduced), which is also advantageous by allowing a larger portion of the OCT scan to be used for visualizing the tissue. Our experience indicates that the adhesion between the contacting glass and the reference layer often combines features of viscous (threshold-less) and dry (threshold-type) friction, so that simple arguments based on invariant mechanical properties for a given layer thickness/ width ratio may be inapplicable. Further, without knowing detailed input information about the relevant stiction characteristics, numerical simulations will not correct this. In such practical cases, direct during-measurement visualization of the strain distribution in the reference layer (and its agreement with the supposed homogeneity) looks rather attractive.

On one hand, verification of the assumed homogeneity of strain over the reference layer thickness may be less critical for visualizing the relative tissue strain distribution. On the other hand, it becomes really important for absolute quantification of the Young modulus, since the influence of adhesion may distort the apparent strain of the reference layer up to several times. Such distortions may be particularly strong for soft silicone sensor layers (with the Young modulus $\sim$ tens of $\mathrm{kPa}$ ) with very small $G / K<10^{-5}$ ratios, and further amplified by rapid compression cycles (e.g., produced by periodic piezo-actuators synchronized with $B$-scan rates often reaching tens or hundreds of $\mathrm{Hz}$ ). Under such conditions, even weak interface adhesion that apparently slightly impedes free lateral expansion of 


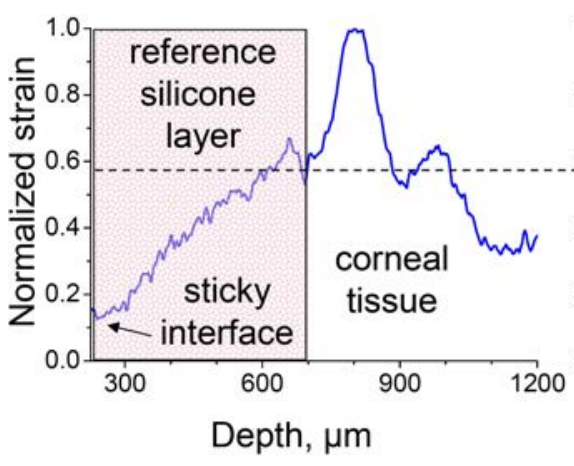

(a)

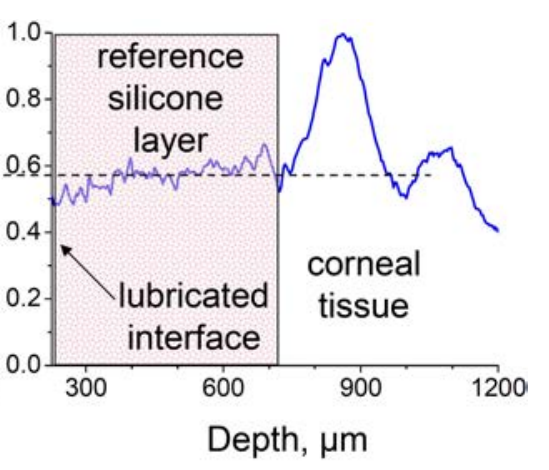

(b)

Fig. 2. Experimental examples of the stiction effect in the reference silicone layer overlaying softer tissue (excised rabbit eye cornea). (a) no lubrication added between the OCT probe and silicone, yielding very pronounced sticking and strain inhomogeneity in silicone; (b) same, but now with a small amount of lubricant (glycerol) added to the left interface. Interestingly that closer to the right silicone-cornea interface (at $\sim 700 \mu \mathrm{m}$ in the figures) and in the tissue itself, the strain contrast profiles are similar for both stiction conditions (the same level of silicone strain near silicone-cornea interface is shown by the dashed line). In contrast, closer to the glass surface, the silicone is very differently deformed in plots (a) and (b).

the reference layer may cause especially significant decrease in its actual axial deformability.

A corresponding experimental example in Fig. 2 demonstrates that the influence of adhesion/stiction at the interface with a rigid surface can reduce deformability of the contacting soft material significantly (several times), which may introduce strong errors in quantification of the elastic modulus of the tissue. Figure 2 shows an example of depth strain profiles across the silicone-tissue sample for two situations: Fig. 2(a) with a normal (sticky) OCR probe-silicone interface, and Fig. 2(b) with lubricant (glycerol) added at the interface. The strain is normalized to its tissue maximum (excised rabbit's cornea). Note that the contrast between the reference-layer strain near the interface with the tissue and strain in the tissue itself are both nearly the same, whereas the local strain in the silicone reference layer may be strongly heterogeneous depending on its stiction to the OCT probe surface. Thus, simplified strain estimate for the entire silicone layer thickness may be in error (as much as $2 \times$ ). Generally, strain visualization within the reference layer helps to evaluate the distorting influence of adhesion/stiction, and will help to assess the various additional methods for minimizing these distortions.

\subsection{Distorting effect of tissue surface curvature}

Another important issue that significantly complicates practical realization of compressional OCE is

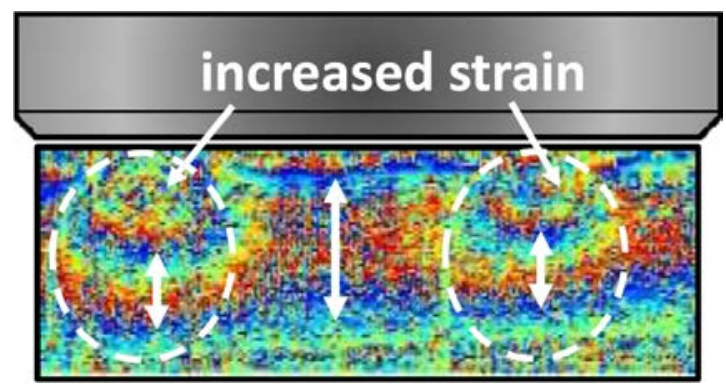

Fig. 3. An example of inhomogeneous strain distribution in a homogeneous silicone sample with an uneven surface (two protrusions). The dotted contours show the regions below the protrusions, where the phase gradient (and, therefore, strain) is significantly greater than in the central fairly flat region (the vertical arrows show the difference in the characteristic scales of the phase-variation periodicity between these regions).

the uneven surface of real tissues. Figure 3 shows an experimental example of such strain heterogeneity for a homogeneous silicone sample with a pair of wavy protrusions at the surface produced when cutting the sample. Below these protrusions, regions of locally increased strain (shown by dotted contours) are clearly seen.

Tissues often have pronounced curvature of the surface as shown in the experimental real-tissue structural image, Fig. 4(a), where under the silicone reference layer, the tissue (a tumor growing in a mouse's ear) has a pronouncedly curved surface. If the rigid OCT probe was directly used to compress the tissue with its surface undulations, without the 


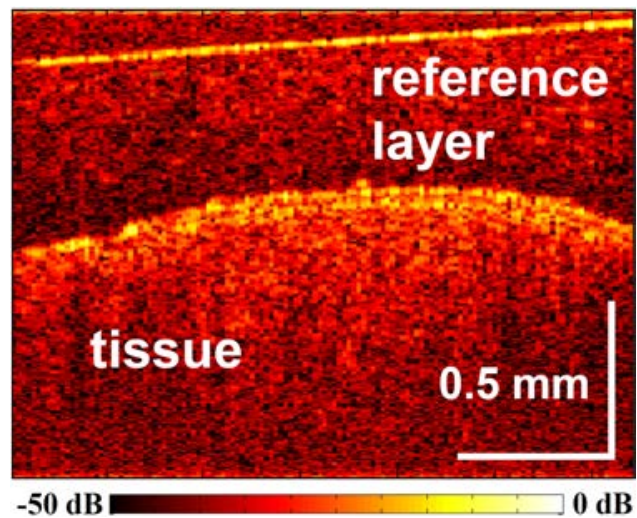

(a)

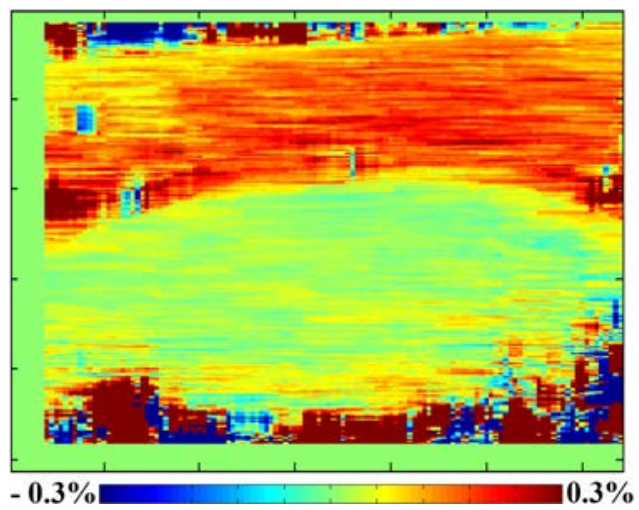

(c)

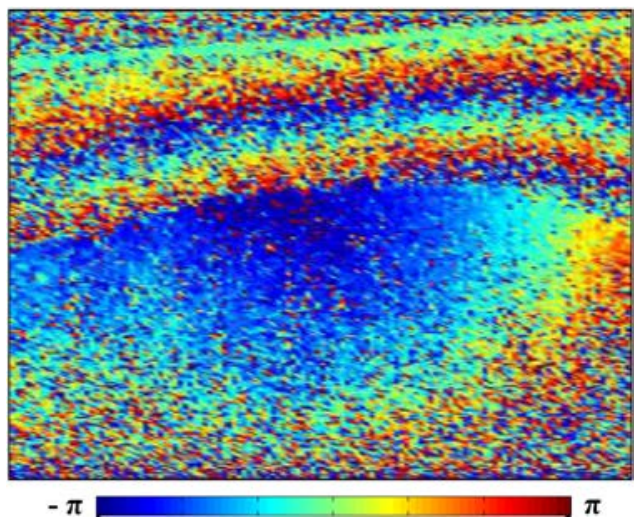

(b)

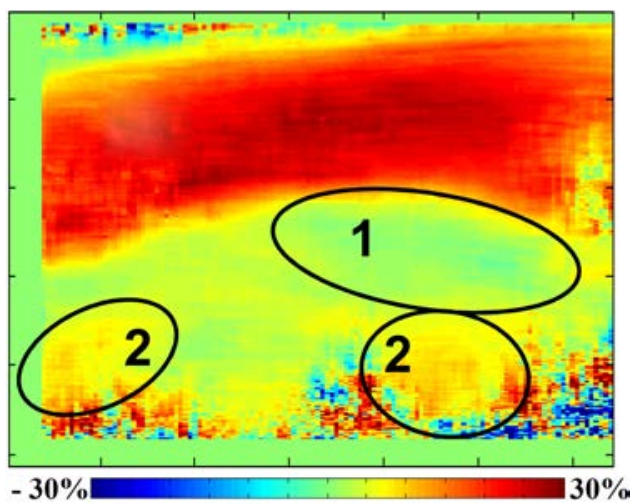

(d)

Fig. 4. Use of a translucent reference sensor layer for OCE imaging of tissue with uneven surface and inhomogeneous distribution of stiffness (a tumor inoculated in a mouse ear): (a) structural OCT image showing the flat probe-sensor layer interface, homogenous and deformed sensor layer, and underlying tissue with an uneven surface (protruding tumor). Contours 1 and 2 mark the tumor region and underlying normal tissue, respectively, (b) color map of phase difference between a reference and deformed frames; note the regular phase variation in the reference layer (indicative of its homogeneity) and irregular phase behavior in the underlying heterogeneous tissue, (c) corresponding map of interframe strain, and (d) cumulative strain accumulated over 130 frames during monotonic compression. Both (c) and (d) exhibit higher strains in the silicone layer (as expected), and lower/heterogenous strains in tissue; cumulative strain maps such as (d) are able to better visualize lower-strain tumor region 1 (encircled) and higher-strain regions 2 (encircled) of softer nearly normal tissue beneath the tumor.

use of the intervening silicone reference layer as in Fig. 4(a), then compression-induced strain could become strongly heterogeneous with maxima near tissue surface protrusions and minima in the valleys as in Fig. 3. In contrast to a priori known homogeneous structure of silicone in Fig. 3, the tissue has unknown stiffness distribution and its surface is not perfectly flat. In practice, even common tissue protrusions/indentations as small as $\sim 10 \mu \mathrm{m}$ may already produce significant distortions of strain when compressed by a flat glass window making the visualized strain heterogeneity to be very difficult to interpret correctly.

Fortunately, a soft and flat intervening reference layer between the OCT probe and tissue can efficiently help to smoothen the distribution of the stress and to counter the effects of tissue surface unevenness. This is demonstrated in Fig. 4 that shows a real-tissue structural image, Fig. 4(a), interframe phase variation (Fig. 4(b) similar to that in Fig. 1(b)) and the corresponding interframe strain, Fig. 4(c), and cumulative strain, Fig. 4(d), corresponding to summation of $\sim 130$ interframe strains obtained during manually-produced monotonic compression. The strain maps, Figs. 4(c) and 4(d), demonstrate that the strain in the center of the scan is fairly uniform in the lateral direction despite the pronounced curvature of the tissue (a tumor inoculated in a mouse ear) located beneath the intervening soft silicone layer. Direct compression of 
the tissue by a rigid surface would produce strongly increased strain (similar to local strain enhancements in Fig. 3) near the top of the tumor, although the latter is actually stiffer and less deformable than the normal tissue beneath it. The strain maps in Figs. 4(c) (interframe strain) and 4(d) (cumulative strain) demonstrate that the soft reference layer indeed helps to create fairly uniform uniaxial stress, so that the strain heterogeneity in the tissue readily delineates the stiffer lower-strain region of the tumor (label 1) and more deformable higher-strain normal tissue beneath it (label 2).

The normal tissue (region 2) is $\sim 3-4 \times$ stiffer than the reference layer, whereas in the tumor, it is stiffer still (perhaps an order of magnitude stiffer than the reference layer). The tumor region is so stiff compared with the reference silicone that the reference layer even experiences apparent stiffening due to adhesion/stiction effects both near the glass and near the interface of the silicone with the stiff region 1. Indeed, Fig. 4(d) shows that the resultant reduction in the reference-layer strain occurs not only near the glass window of the OCT probe, but also above the very stiff tumor region 1 . This effect should be clearly understood when quantifying the stiffness of very stiff regions below the reference layer, since even for fairly accurately measures of strains both in the tissue and the reference layer, the apparent stiffening of the latter due to stiction may notably distort the actual stiffness contrast.

\subsection{Enhancing SNR in compressional OCE when periodic averaging is impossible}

Another practical consideration in compressional OCE is its robustness with respect to noises and ability to obtain useful SNR even if its enhancement via periodic averaging is impossible. The latter feature is especially important for in vivo deployment using manually-produced straining of the studied region, because various natural motions of the tissue (influence of breathing, heart/blood pressure beats, etc.) strongly reduce the potential utility of stable periodic actuators. Thus, for in vivo measurements (but also for studying ex vivo samples), the possibility to work without additional auxiliary actuators and periodic averaging is very attractive.
In this context, the ability of the "vector" method $^{31}$ to estimate phase-gradient within a chosen processing window without the necessity of conventionally used phase-unwrapping procedures is advantageous. For this to succeed, the strain should not cause the phase wrapping between vertically adjacent pixels, but this limitation is not very restricting; thus, the method is applicable throughout the entire practical/interesting strain range (up to $\sim 1 \%$ ), for which there is not yet very strong decorrelation of the compared images. The possibility to process interframe phase differences without the necessity to unwrap multiple-period phase wrapping (which is known to be an errorprone procedure) enables the use of higher interframe strains (up to $\sim 10^{-2}$ ) that are larger than conventionally used strains $10^{-4}-10^{-3}$ in phaseresolved methods. ${ }^{32}$ This is favorable for enhancing the effective SNR, because the resultant elevated phase gradients can be estimated with better relative accuracy than weaker gradients that are often masked by various measurement noises (and thus necessitate periodic averaging, if possible ${ }^{18}$ ). Indeed, for a chosen scale $L_{z}$ over which the gradient is estimated (i.e., vertical size of the processing window), the gradient $d(\delta \varphi) / d z$ of the interframe phase difference and the total phase variation $\Delta(\delta \varphi)$ over the processing window are proportional to each other, $L_{z} \cdot d(\delta \varphi) / d z=\Delta(\delta \varphi)$. Thus, for a given uncertainty in measuring phase $\pm \sigma_{\varphi}$, the effective SNR for estimating phase gradient $d(\delta \varphi) / d z$ is determined by the ratio $\Delta(\delta \varphi) / \sigma_{\varphi} \propto d(\delta \varphi) / d z$. In other words, effective SNR for determining phase gradient is proportional to the phase gradient itself and, therefore, local strain. This stronger-strain detection advantage is diagrammatically illustrated in Fig. 5.

Figure 5(a) shows schematic one-dimensional (1D) fragments of interframe phase-difference dependencies on the depth for three different strain levels with exactly the same numerically added noise that almost completely masks the regular slope of the dependence for the smallest strain, but for the higher strains, the slopes look much less noisy. Plots Figs. 5(b) and 5(d) show rigorously simulated (using model of Ref. 33) 2D phase-variation diagrams for strains $\varepsilon \sim 10^{-3}$ and $\varepsilon \sim 5 \cdot 10^{-3}$ in a two-layer sample with $50 \%$ contrast in stiffness. The simulation parameters correspond to a typical OCT system operating at $1300 \mathrm{~nm}$ with spectral width $\sim 100 \mathrm{~nm}$, and the "decorrelation noise" due 


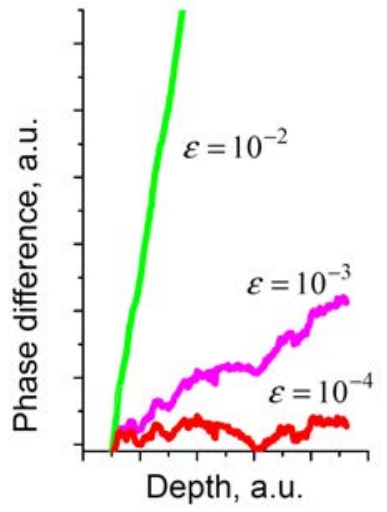

(a)

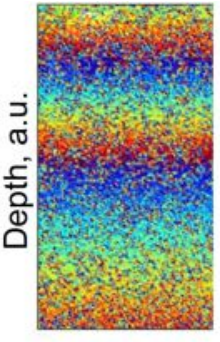

(b)

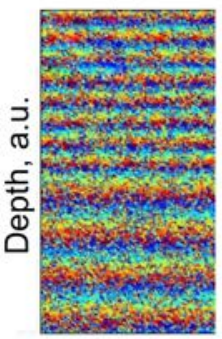

(d)

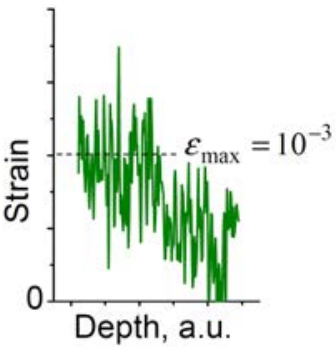

(c)

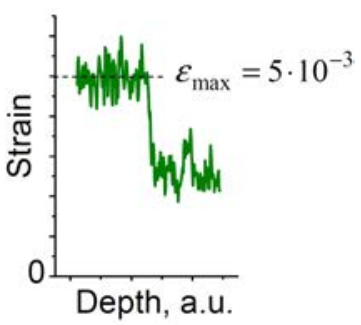

(e)

Fig. 5. Increased strain (but still below the onset of very strong decorrelation) is favorable for increasing effective SNR. The three curves in 1D schematic plot show (a) interframe phase difference as a function of depth for three different strains (phase-difference slopes), but exactly the same numerically added noise, the masking influence of which significantly reduces with moderately increasing strain. Plots (b) and (d) show rigorously simulated (using model of Ref. 33) 2D phase-variation maps for maximal strains $\varepsilon=10^{-3}$ and $\varepsilon=5 \cdot 10^{-3}$ for a two-layer tissue with $50 \%$ contrast in stiffness for the same level of the added noise (SNR $4.5 \mathrm{~dB}$ ). Panels (c) and (e) are the corresponding vertical strain profiles to show that the effective SNR for finding strain map is significantly better for $\varepsilon=5 \cdot 10^{-3}$ than that for $\varepsilon=10^{-3}$.

to displacements of scatterers is also reproduced in the simulation, although it is not yet very significant for such strains. The additional measurement noises (with $\mathrm{SNR} \sim 4.5 \mathrm{~dB}$ ) are of the same level in both cases. For $\varepsilon \sim 10^{-3}$, such noise very strongly impedes determining the phase-variation gradient (even for the used noise-tolerant "vector method" ${ }^{31}$ ) as shown in Fig. 5(c). In contrast, for modestly greater strain $\varepsilon \sim 5 \cdot 10^{-3}$, the phase-gradients (i.e., local strains) exhibit higher effective SNR, so that the reconstructed strain profile clearly reveals the stiffness contrast between the layers as demonstrated in Fig. 5(e).

In addition to this increased noise robustness due to using moderately higher interframe strains and applying the vector method, further SNR enhancement can be obtained by finding cumulative strains via summation of interframe strains for a sequence of frames; such a cumulative-strain map has already been presented in Fig. 4(d). The monotonic tissue loading in this example was manually produced. In comparison with interframe strain, Fig. 4(c), the cumulative-strain map, Fig. 4(d), is significantly less noisy and clearly reveals details (e.g., the softer region 2 beneath the tumor) that are hardly distinguishable in the interframe strain map Fig. 4(c).

\subsection{Influence of tissue nonlinearity on evaluation of elastic modulus in $O C E$}

Cumulative strain approach for enhancing SNR in a nonperiodic-deformation scenario makes it possible to significantly extend the range of visualized strains to a several percent level (and even great$\mathrm{er}^{34,35}$ ), compared to sub-percentage levels common in interframe phase-resolved measurements. ${ }^{29}$ Such an extended strain range opens the possibility to study possible deviation of the tissue response from the linear Hooke's law (assumed in Eq. (1)), enabling the characterization of the nonlinear behavior of elastic modulus in compressional OCE.

In agreement with previous data based on macroscopic mechanical measurements ${ }^{36,37}$ and measurements based on ultrasound technique, ${ }^{38}$ preliminary OCE results ${ }^{21,22}$ indicate that soft biological tissues may indeed exhibit notable nonlinearity for strains $\sim 10-20 \%$, but also for smaller strains. Silicones also begin to exhibit notable 
deviations from the linear behavior for strains $>20$ $30 \% .{ }^{17}$ However, the reference silicone layer quite often has elastic modulus several times greater than tissue and still exhibits linear behavior whereas soft tissue may already demonstrate nonlinear response. Thus, plotting the tissue strain against the reference-layer strain in compressional OCE with properly chosen stiffness of the reference layer corresponds to plotting the stress-strain relationship of the tissue including the nonlinear regime, which opens up an exciting possibility for studying tissue response over a wide strain range, including characterization of its potentially nonlinear behavior. This may be useful in distinguishing different tumor types based on their nonlinear-elastic response differences. Some initial demonstrations of this nonlinearity approach using ultrasound have been reported, ${ }^{38}$ using iterative matching of the measured and predicted deformations for nearly incompressible material with assumed VerondaWestman constitutive nonlinearity law; ${ }^{39}$ such methods are yet to be attempted in compressional OCE, and may indeed prove useful. In contrast, our proposed methodology is computationally simpler and does not require a priori assumptions about the character of the tissue nonlinearity. For the derived nonlinearity, one can use a reasonable approximating function (e.g., add a simple quadratic-instrain correction to the linear Hooke's law or choose a more sophisticated dependence) and determine the fitting parameters including nonlinear one(s).

An experimental example of a so-obtained stressstrain relationship is presented in Fig. 6 that demonstrates a pronounced, but intuitively unexpected, nonlinearity in the explored rather modest strain range $(<2.5 \%)$. These studies of human tissue (breast cancer tissue excised during mastectomy) were carried with informed patient consent and approved by the Ethics Committee of the Nizhny Novgorod State Medical Academy. Despite apparently small straining of the tissue (much smaller than $10-30 \%$, where nonlinearity is usually expected), Fig. 6 clearly demonstrates that the elastic modulus of the tissue (i.e., derivative $\partial \sigma / \partial \varepsilon$ ) varies by $\sim 3 \times$ for $\varepsilon<2.5 \%$. As pre-straining of tissue in the order of a few percent is often required in OCE (e.g., to ensure good probe-tissue contact, especially in the common cases when the latter is not ideally flat), this implies that the elastic modulus "result" one obtains will be very much experiment-dependent. Even if periodic averaging can be used by

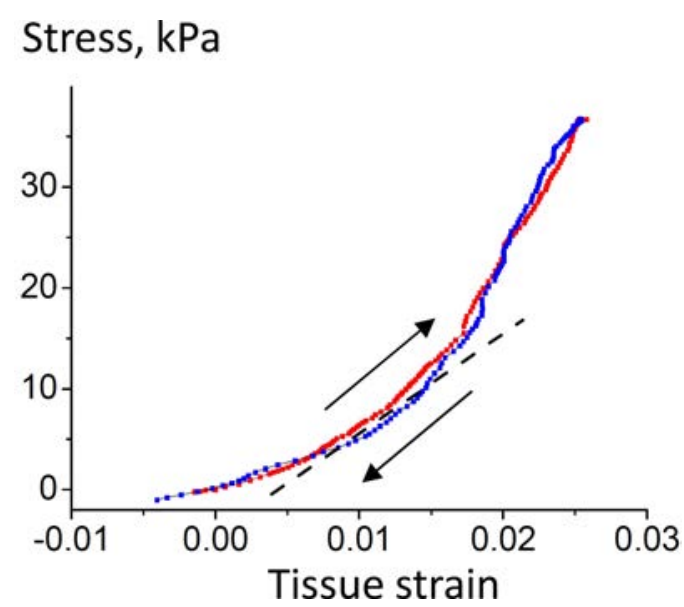

Fig. 6. Experimental data showing nonlinear-elastic stressstrain behavior obtained using compressional OCE, with a silicone reference layer overlying an excised breast cancer tissue sample. Note that the tissue Young modulus $\partial \sigma / \partial \varepsilon$ varies greatly (range $\sim 400-1400 \mathrm{kPa}$ ), although the strain range is modest $<2.5 \%$. The slope of the dashed line corresponds to the mid-range Young modulus value of $\sim 1000 \mathrm{kPa}$. For comparison, the stiffer silicone reference layer had $E \sim 200 \mathrm{kPa}$. Arrows indicate loading (upward) and unloading (downward) tissue response; their near-overlap shows no hysteresis in this breast cancer tissue (for details, see text).

applying small oscillating strain with smaller amplitudes $\varepsilon<10^{-3}$ typical for phase-sensitive OCE, the estimated elastic modulus may still differ several times because of initial pre-straining. Thus, even in apparently "small strain" elastographic measurements, potential strong influence of the tissue nonlinearity should be taken into account. The bidirectional loading-unloading curves shown in Fig. 6 overlap, indicating no hysteresis for this breast cancer sample; however, we have preliminary data (manuscript in preparation) that many other tissue types do exhibit mechanical hysteresis, further complicating the nonlinear tissue response. Overall then, compressional OCE utilizing an intervening reference layer opens up a novel for OCE opportunity to study nonlinear tissue characteristics that may offer additional valuable biophysical insights.

\section{Summary and Conclusions}

Although the basic idea of compressional OCTbased elastography ${ }^{16}$ looks quite straightforward, its realization was nontrivial and it took about 15 years to develop methods of reasonably accurate strain mapping that enabled visualization of 
relative distribution of stiffness. In the last several years, significant progress was achieved in solving another challenging problem in compressional OCE, that of absolute quantification of the tissue stiffness. Although the basic idea of using sensor (reference) layers with known stiffness also appears quite simple, in this paper, we point out some obstacles arising in its practical use and indicate possible ways of overcoming these difficulties.

In particular, adhesion (stiction) of the calibration layer to the OCT probe surface, as well as curvature and/or protrusion of the tissue surface both significantly distort spatial strain distribution and complicate the derivation of the true tissue stiffness maps. These distortions should be taken into account in compressional OCE of biological tissues, and potentially mitigated as suggested in the current paper. In in vivo applications of OCE, the use of periodic averaging for enhancing SNR may not be feasible, and we discuss the use of the "vector" method with cumulative strain mapping as a possible route for maintaining suitable SNR levels.

Finally, even if the above-mentioned methodological problems are resolved, conventional stiffness quantification in compressional OCE based on linear paradigm of tissue elasticity may prove inadequate because of intrinsic elastic nonlinearity of biological tissues. The nonlinear effects may manifest even for small strains (in the order of several percent, where linear behavior is intuitively expected). Recently developed realizations of compressional OCE enable measurements of this complex nonlinear behavior, opening up interesting prospects for characterizations of elastic properties of tissues beyond the linear approximation with potential novel diagnostic possibilities.

\section{Acknowledgments}

The study has been supported by the Russian Federation Government contract No. 14.B25.31. 0015. Signal processing development was supported by the Russian Foundation for Basic Research Grant No. 16-02-00642 and Russian President grant for young scientists No. MK-6504.2016.2. Authors are grateful to Drs. David Sampson and Brendan Kennedy from the University of Western Australia for their very helpful expertise sharing in the preparation/utilization of silicone sensor layers.

\section{References}

1. K. J. Parker, M. M. Doyley, D. J. Rubens, "Imaging the elastic properties of tissue: The 20 year perspective," Phys. Med. Biol. 56(1), R1-R29 (2011).

2. L. D. Landau, E. M. Lifshitz, Theory of Elasticity, Course of Theoretical Physics, Elsevier, Amsterdam (1986).

3. X. Liang, V. Crecea, S. A. Boppart, "Dynamic optical coherence elastography: A review," J. Innov. Opt. Health Sci. 3(04), 221-233 (2010).

4. C. Sun, B. Standish, V. X. D. Yang, "Optical coherence elastography: Current status and future applications," J. Biomed. Opt. 16(4), 043001 (2011).

5. B. F. Kennedy, K. M. Kennedy, D. D. Sampson, "A review of optical coherence elastography: Fundamentals, techniques and prospects," IEEE J. Sel. Top. Quantum Electron. 20, 7101217 (2014).

6. L. A. Matveev, V. Y. Zaitsev, A. L. Matveev, G. V. Gelikonov, V. M. Gelikonov, A. Vitkin, "Novel methods for elasticity characterization using optical coherence tomography: Brief review and future prospects," Photonics Lasers Med. 3(4), 295-309 (2014).

7. S. Wang, K. V. Larin, "Optical coherence elastography for tissue characterization: A review," J. Biophotonics 8(4), 279-302 (2015).

8. K. V. Larin, D. D. Sampson, "Optical coherence elastography-OCT at work in tissue biomechanics," Biomed. Opt. Express 8(2), 1172-1202 (2017).

9. B. F. Kennedy, P. Wijesinghe, D. D. Sampson, "The emergence of optical elastography in biomedicine," Nat. Photonics 11(4), 215-221 (2017).

10. S. Wang, K. V. Larin, J. Li, S. Vantipalli, R. K. Manapuram, S. Aglyamov, S. Emelianov, M. D. Twa, "A focused air-pulse system for optical-coherence-tomography-based measurements of tissue elasticity," Laser Phys. Lett. 10(7), 075605 (2013).

11. J. Li, S. Wang, M. Singh, S. Aglyamov, S. Emelianov, M. D. Twa, K. V. Larin, "Air-pulse OCE for assessment of age-related changes in mouse cornea in vivo," Laser Phys. Lett. 11(6), 065601 (2014).

12. M. D. Twa, J. Li, S. Vantipalli, M. Singh, S. Aglyamov, S. Emelianov, K. V. Larin, "Spatial characterization of corneal biomechanical properties with optical coherence elastography after UV crosslinking," Biomed. Opt. Express 5(5), 14191427 (2014).

13. N. Le, S. Song, G. Nabi, R. Wang, Z. Huang, "Quantitative measurement and real-time tracking of high intensity focused ultrasound using phasesensitive optical coherence tomography: Feasibility study," Int. J. Hyperthermia 32(6), 713-722 (2016). 
14. J. Zhu, Y. Qu, T. Ma, R. Li, Y. Du, S. Huang, K. K. Shung, Q. Zhou, Z. Chen, "Imaging and characterizing shear wave and shear modulus under orthogonal acoustic radiation force excitation using OCT Doppler variance method," Opt. Lett. 40, 20992102 (2015).

15. C. Li, G. Guan, Y. Ling, Y. Hsu, S. Song, J. T. Huang, S. Lang, R. K. Wang, Z. Huang, G. Nabi, "Detection and characterization of biopsy tissue using quantitative optical coherence elastography (OCE) in men with suspected prostate cancer," Cancer Lett. 357(1), 121-128 (2015).

16. J. M. Schmitt, "OCT elastography: Imaging microscopic deformation and strain of tissue," Opt. Express 3(6), 199-211 (1998).

17. K. M. Kennedy, L. Chin, R. A. McLaughlin, B. Latham, C. M. Saunders, D. D. Sampson, B. F. Kennedy, "Quantitative micro-elastography: Imaging of tissue elasticity using compression optical coherence elastography," Sci. Rep. 5, 15538 (2015).

18. W. M. Allen, L. Chin, P. Wijesinghe, R. W. Kirk, B. Latham, D. D. Sampson, C. M. Saunders, B. F. Kennedy, "Wide-field optical coherence micro-elastography for intraoperative assessment of human breast cancer margins," Biomed. Opt. Express 7(10), 4139 (2016).

19. V. Y. Zaitsev, A. L. Matveyev, L. A. Matveev, G. V. Gelikonov, V. M. Gelikonov, A. Vitkin, "Deformation-induced speckle-pattern evolution and feasibility of correlational speckle tracking in optical coherence elastography," J. Biomed. Opt. $\mathbf{2 0}(7), 075006$ (2015).

20. M. M. Doyley, "Model-based elastography: A survey of approaches to the inverse elasticity problem," Phys. Med. Biol. 57(3), R35-R73 (2012).

21. W. Allen, P. Wijesinghe, K. Kennedy, L. Chin, D. Sampson, B. Kennedy, "Quantifying tissue stiffness and the effect of nonlinearity using compression optical coherence elastography," Front. Opt. OSA Technical Digest (online) (Optical Society of America, 2015), Paper FW4E. 7.

22. Y. Qiu, F. R. Z. Zaki, N. Chandra, S. A. Chester, $\mathrm{X}$. Liu, "Nonlinear characterization of elasticity using quantitative optical coherence elastography," Biomed. Opt. Express 7(11), 4702-4710 (2016).

23. L. Dong, P. Wijesinghe, J. T. Dantuono, D. D. Sampson, P. R. T. Munro, B. F. Kennedy, A. A. Oberai, "Quantitative compression optical coherence elastography as an inverse elasticity problem," IEEE J. Sel. Top. Quantum Electron. 22(3), 277287 (2016).

24. P. Wijesinghe, D. D. Sampson, B. F. Kennedy, "Computational optical palpation: A finite-element approach to microscale tactile imaging using a compliant sensor," J. R. Soc. Interface 14, 20160878 (2017).

25. G. W. C. Kaye, T. H. Laby, Tables of Physical and Chemical Constants, 15th edition. Longman, London (1993).

26. K. L. Johnson, Contact Mechanics, Cambridge University Press, Cambridge (1987).

27. J. Ophir, I. Cespedes, H. Ponnekanti, Y. Yazdi, X. Li, "Elastography: A quantitative method for imaging the elasticity of biological tissues," Ultrason. Imaging 13, 111-134 (1991).

28. K. M. Kennedy, S. Es'haghian, L. Chin, R. A. McLaughlin, D. D. Sampson, B. F. Kennedy, "Optical palpation: Optical coherence tomographybased tactile imaging using a compliant sensor," Opt. Lett. 39(10), 3014-3017 (2014).

29. B. F. Kennedy, S. H. Koh, R. A. McLaughlin, K. M. Kennedy, P. R. T. Munro, D. D. Sampson, "Strain estimation in phase-sensitive optical coherence elastography," Biomed. Opt. Express 3, 1865-1879 (2012).

30. V. Y. Zaitsev, A. L. Matveyev, L. A. Matveev, G. V. Gelikonov, E. V. Gubarkova, N. D. Gladkova, A. Vitkin, "Hybrid method of strain estimation in optical coherence elastography using combined subwavelength phase measurements and supra-pixel displacement tracking," J. Biophotonics 9(5), 499509 (2016).

31. V. Y. Zaitsev, A. L. Matveyev, L. A. Matveev, G. V. Gelikonov, A. A. Sovetsky, A. Vitkin, "Optimized phase gradient measurements and phase-amplitude interplay in optical coherence elastography," J. Biomed. Opt. 21(11), 116005 (2016).

32. L. Chin, A. Curatolo, B. F. Kennedy, B. J. Doyle, P. R. T. Munro, R. A. McLaughlin, D. D. Sampson, "Analysis of image formation in optical coherence elastography using a multiphysics approach," Biomed. Opt. Express 5(9), 2913 (2014).

33. V. Y. Zaitsev, L. A. Matveev, A. L. Matveyev, G. V. Gelikonov, V. M. Gelikonov, "A model for simulating speckle-pattern evolution based on close to reality procedures used in spectral-domain OCT," Laser Phys. Lett. 11(10), 105601 (2014).

34. V. Y. Zaitsev, A. L. Matveyev, L. A. Matveev, G. V. Gelikonov, A. I. Omelchenko, D. V. Shabanov, O. I. Baum, V. M. Svistushkin, E. N. Sobol, "Optical coherence tomography for visualizing transient strains and measuring large deformations in laserinduced tissue reshaping," Laser Phys. Lett. 13(11), 115603 (2016).

35. V. Y. Zaitsev, A. L. Matveyev, L. A. Matveev, G. V. Gelikonov, A. I. Omelchenko, O. I. Baum, V. Gelikonov, S. E. Avetisov, A. V. Bolshunov, V. I. Siplivy, D. V. Shabanov, A. Vitkin, E. N. Sobol, 
"Optical coherence elastography for strain dynamics measurements in laser correction of cornea shape," J. Biophotonics 14, 1-15 (2017), http://doi.org/ 10.1002/jbio.201600291.

36. F. A. Duck, Physical Properties of Tissues - $A$ Comprehensive Reference Book, Academic Press, Sheffield, UK (1990).

37. A. Delalleau, G. Josse, J.-M. Lagarde, H. Zahouani, J.-M. Bergheau, "A nonlinear elastic behavior to identify the mechanical parameters of human skin in vivo," Skin Res. Technol. 14(2), 152-164 (2008).

38. A. A. Oberai, N. H. Gokhale, G. Sevan, P. E. Barbone, T. J. Hall, A. M. Sommer, J. Jiang, "Linear and nonlinear elasticity imaging of soft tissue in vivo: Demonstration of feasibility," Phys. Med. Biol. 54, 1191-1207 (2009).

39. D. R. Veronda and R. A. Westman, "Mechanical characterization of skin — finite deformations," J. Biomech. 3, 111-122 (1970). 